\title{
Extracellular vesicles in blood: are they viable as diagnostic and predictive tools in breast cancer?
}

\section{Róisín Daly ${ }^{1,2,3}$ and Lorraine O'Driscoll ${ }^{1,2,3}$}

\footnotetext{
${ }^{1}$ School of Pharmacy and Pharmaceutical Sciences, Trinity College Dublin, Ireland

${ }^{2}$ Trinity Biomedical Sciences Institute, Trinity College Dublin, Ireland

${ }^{3}$ Trinity St. James's Cancer Institute, Trinity College Dublin, Ireland
}

Extracellular vesicles (EVs), often described as mini-maps of their cells of origin, are found in the bloodstream and can be rich sources of cargo released from cancer cells. As such, they could be collected through minimally invasive methods and potentially used as biomarkers. However, the relatively complicated methodologies that separate the purest EVs are the least likely to be translated to the clinic, whereas simpler methods are non-selective for EVs. Notwithstanding this, research is underway to identify blood-based EV-associated diagnostic and predictive biomarkers for breast cancer. There is reason to be optimistic that some approaches will yield useful biomarkers. Thus, further studies with larger cohorts of appropriate samples are warranted.

\section{Introduction}

Breast cancer (BC) is the most common cancer in women (World Health Organization, https://www.who.int/cancer/prevention/ diagnosis-screening/breast-cancer/en/), and approximately $1 \%$ of BCs occur in men. More than 1.7 million new cases of BC are diagnosed worldwide annually, and every woman has at least a one in eight chance of developing BC. If the cancer is detected while still localised in the breast, the 5 -year survival rate is up to $98 \%$, but if it has metastasised, the survival rate drops to $26 \%$ [1] (American Cancer Society, https://www.cancer.org/cancer/ breast-cancer/about/how-common-is-breast-cancer.html). Early detection and optimal treatment are key to survival. However, diagnosis still often occurs by chance and not early enough. Often the diagnosis is made when a growth or irregularity is detectable and is subsequently confirmed by biopsy.

Minimally invasive blood-based diagnostic markers would be invaluable. After diagnosis, tissue-based biomarkers such as human epidermal growth factor receptor 2 (HER2), oestrogen receptor (ER) and Ki67 are useful for determining prognosis and guiding choices of treatment. However, drug-resistance, whether innate or acquired, is a major clinical problem that is compounded by the lack of

Corresponding author. O’Driscoll, L. (lodrisc@tcd.ie) predictive biomarkers (i.e., companion diagnostics) [2]. This has serious implications for patients' quality-of-life and longevity. Furthermore, the costs of many anticancer treatment regimens are substantial, and their appropriate use is essential for societal and economic benefit. Thus, efforts are underway to determine the potential of blood-based extracellular vesicles (EVs) as diagnostic and predictive biomarkers (Fig. 1). Also, for readers who may not be familiar with BC, Table 1 summarises some key terms used here.

The term exosome dates back approximately 50 years, when it was used in relation to the fruit fly (Drosophila) [3]. Some 20 years later, seminal papers appeared reporting that exosomes were involved in transporting transferrin receptor from cells during reticulocyte maturation [4]. Since then, interest in exosomes has increased substantially, with more than 2,500 publications in this field listed on PubMed in 2019 alone. The huge interest from academics, clinicians and industry is due to increasing evidence that substantial cargos of information are released from cells via these lipid-bilayer-enclosed vesicles, typically termed exosomes and microvesicles, and that these have both physiological and pathophysiological relevance. These vesicles are proposed to be tailor-made mini-maps of their cell of origin that are transported in the bloodstream and other body fluids, and that are involved in cell-to-cell communication [5]. 


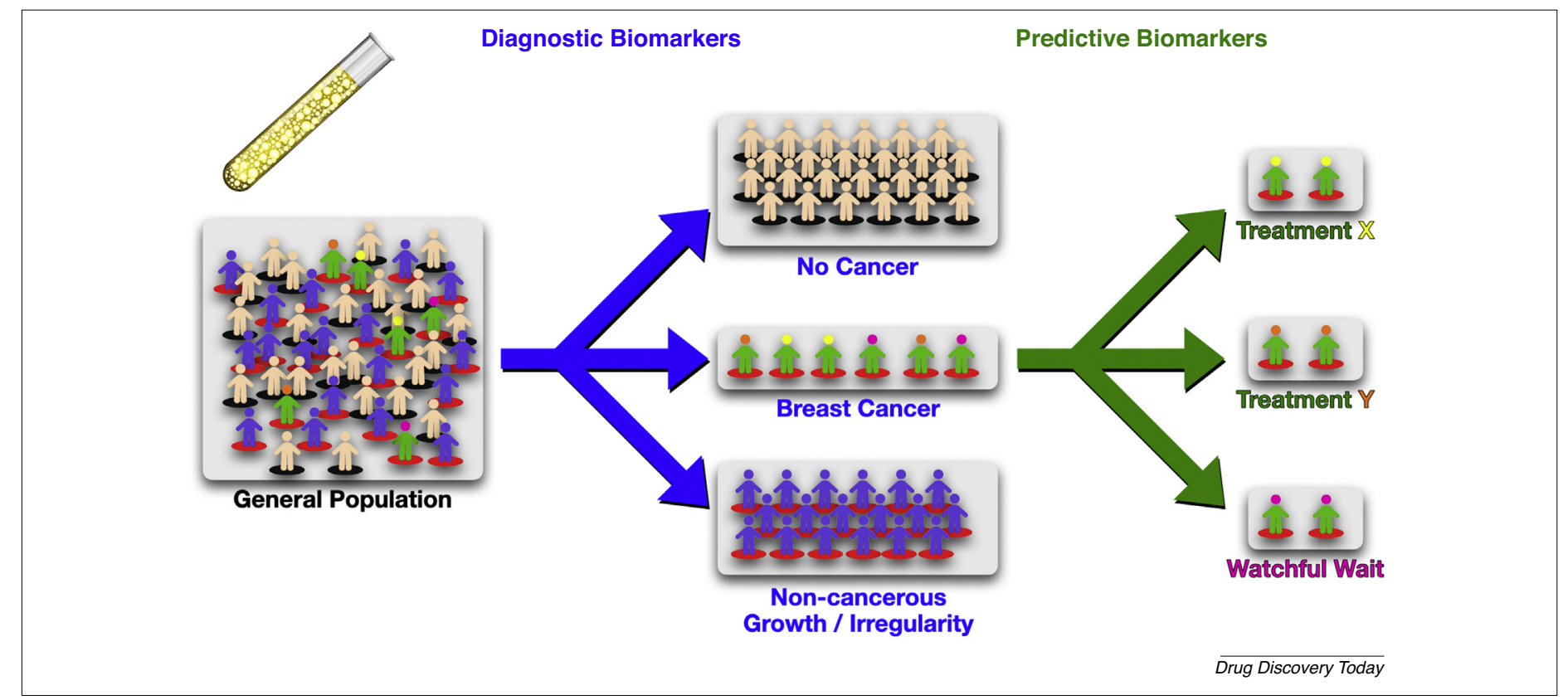

Predictive Biomarkers

\section{FIGURE 1}

Extracellular vesicles as diagnostics and predictive biomarkers. Minimally invasive blood-based biomarkers could identify those who have breast cancer from those who are healthy with no cancer, including those who have a benign breast growth or similar irregularity. For those who are diagnosed with breast cancer, predictive biomarkers would aid selection of the optimal treatment for the individual, if treatment is necessary (for some, watchful waiting might be more appropriate than intervention).

Exosomes and microvesicles, collectively termed EVs, were originally classified into subgroups on the basis of their size and cellular origin (exosomes are approximately $30-120 \mathrm{~nm}$ and of endosomal origin, whereas microvesicles are $120-1000 \mathrm{~nm}$ and bud from the cell membrane) [6]. A limited number of authors propose that exosomes also bud from the cell membrane, although this is not the general consensus [7]. Adding to the nomenclature's complexity, some reports use alternative terms, including ectosomes, microparticles, oncosomes and prostasomes. All of these are EVs. However, once EVs have been released from cells into the environment (e.g., into blood), we cannot be certain whether they originated from the cells' endosomal pathway or directly from the cell membrane [8]. Furthermore, EV size distinctions are not absolute. Arguably, for cancer, what is more important than the exact size or route of cellular exit of EVs is their potential as minimally-invasive biomarkers.

In 2018, an important milestone was reached regarding methodologies for EV separation/isolation and analysis as biomarkers (or, indeed, for any purpose). Appreciating that absolute purification of EVs from any biofluid is an unrealistic goal, 382 members of

TABLE 1

\section{Terminology related to breast cancer used in this review}

Term Explanation

LCIS, ILC, DCIS, IDC These terms are used to explain the location of the abnormal cells within the breast:

- Lobular carcinoma in situ (LCIS) is when abnormal cells are found in the milk-producing glands (lobules) of the breast, but those cells are contained within the lobules

- Invasive lobular carcinoma (ILC) is BC that begins in the lobules of the breast, but which invades beyond the lobule

- Ductal carcinoma in situ (DCIS) is when abnormal cells are found in the lining of the milk ducts (i.e., the ducts that carry breast milk to the nipple)

- Invasive ductal carcinoma (IDC) is BC that starts in cells that line the milk ducts, but which invades beyond the duct. Of note, IDC accounts for approximately $80 \%$ of all invasive BC in women, and approximately $90 \%$ of all invasive $B C$ in men.

HER2, ER, HR ${ }^{+}$TNBC $B C$ can be subtyped on the basis of some cell surface proteins. This subtyping helps in selecting pharmacological treatments, and includes:

- Human epidermal growth factor receptor 2 (HER2)-overexpressing BC. These BCs are candidates for treatment with HER2targeting drugs, such as trastuzumab and lapatinib

- Oestrogen receptor (ER)-positive BC. These BCs are candidates for treatment with ER-targeting drugs, such as tamoxifen - $\mathrm{HR}^{+} \mathrm{BCs}$ are where the cells are positive for $\mathrm{ER}$ and the progesterone receptor (PR)

- Triple-negative breast cancer (TNBC) does not overexpress HER2, ER or PR. So far, a specific cell surface marker has not been identified as a therapeutic target for TNBC.

Classical chemotherapy, such as docetaxel or paclitaxel, is frequently used as part of the treatment in all cases cancer, including BC, as an indication of the rate of proliferation of the cancer cells 
the EV community published guidelines for appropriate EV isolation and fundamental characterisation. These guidelines seek to provide confidence that what has been isolated are indeed EVs, prior to more advanced characterisation [8]. A worldwide survey concluded that the most common isolation method is ultracentrifugation [9]. Ultracentrifugation-based techniques that involve density gradients are generally accepted to yield the purest, or 'cleanest', EV preparations. For example, a comparative study of four methods reported that density-gradient ultracentrifugation methods outperform kit-based methods such as ExoQuick and Total Exosome Isolation precipitation in terms of purity [10].

However, when considering cancer biomarkers, ultracentrifugation-based methodologies have substantial limitations: they are not high throughput, require an ultracentrifuge and cannot be easily translated to the clinic. A subsequent comparison of EV separation from plasma by ultracentrifugation, ExoQuick, Exospin precipitation and size-exclusion chromatography (SEC) found that precipitation methods provided the least pure preparations, but that SEC was comparable to the ultracentrifugationbased density-gradient method, giving hope that this approach could be suitable for clinical translation [11].

Because lipoproteins (which occur approximately 100-fold more frequently than EVs in plasma) span the size ranges of EVs, separating EVs by ultracentrifugation on a density gradient followed by SEC has been reported to be an optimal way of isolating plasma EVs with high purity [12]. Although this combined approach improves EV purity, it also adds complexity to the procedure. Furthermore, this technique requires procuring a relatively large volume of blood (around $40 \mathrm{ml}$ would be required to obtain up to $12 \mathrm{ml}$ of plasma, as used here) or pooling together a number of samples to prove how relatively pure the isolated EVs are, which is typically not translatable to the clinic. For example, it would not be appropriate to draw large volumes of blood from an already sick cancer patient, who might have numerous blood samples taken over the course of their treatment. More appropriate volumes of serum or plasma for biomarker analysis in such a setting might be in the order of $100 \mu$ l. Therefore, applying extensive methods for EV isolation and fundamental characterisation would not be possible routinely in the clinical setting.

Before deciding on EV-isolation methods for biomarker studies, due consideration should be given to pre-analytical steps. As we reported, because of its high concentration of soluble proteins and lipoproteins, blood has high viscosity and density, which hampers EV concentration, isolation and detection. To achieve rigour and reproducibility, important consideration should be given to, for example, how the blood donor presented (e.g., fasting versus nonfasting), how the blood was drawn, how it was processed to serum or plasma, and how it was stored before EV isolation [13]. Platelets are a rich source of EVs, so platelet-depleted plasma is considered to be the ideal blood product for progressing to EV isolation for biomarker studies.

Of course, it must be recognised that for a candidate molecule (or molecules) to be a potentially useful diagnostic and/or predictive biomarker in blood, it does not necessarily have to be EV cargo. In fact, the relative limited numbers of blood-based biomarkers that form the current standard-of-care do not involve isolating EVs. However, moving forward in this relatively new field of research, if studies are claiming that EVs and/or EV cargo are biomarkers, it is important that necessary effort is invested into providing evidence to support that claim.

\section{EVs as diagnostic biomarkers}

Here, we review studies that evaluate EVs as potential diagnostic biomarkers for BC, typically in chronological order (Table 2). It is noteworthy that although it has been established that ExoQuick (a proprietary precipitating polymer) does not separate EVs from many other precipitates of serum or plasma, it has been the most-used method for this purpose. This is probably because of its ease and speed of use, with no requirement for an ultracentrifuge. However, all RNAs and proteins analysed in such studies cannot be assumed to be EV cargo. It is noteworthy that although all studies in which ExoQuick was used for the discovery of BC diagnostic or predictive biomarkers (see below) seem to have used the original form of ExoQuick, such precipitation methods are evolving. In the future, more advanced methods might be a source of differing and, indeed, improved results. For example, ExoQuick LP (which claims to remove contaminating lipoprotein particles from plasma and serum) and ExoQuick Plasma Prep with Thrombin (which includes a de-fibrinating plasma step prior to isolation) are also available.

Studying ExoQuick precipitates from serum of 44 patients [whose tumour types were described as $n=29$ luminal; $n=3$ HER2; and $n=12$ triple-negative BC (TNBC)] and 12 healthy controls, Eichelser et al. [14] reported EV miR-101 $(p=0.0001)$ and EV miR-372 $(p=0.021)$ to be at higher levels in the isolates of BC samples with compared with controls. EV miR-373 levels did not differ significantly between all of the represented BC subtypes when collectively compared to controls, but were higher in TNBC when compared with luminal BC $(p=0.027)$ and healthy controls $(p=0.001)$. Although this study shows promise, the numbers of samples being compared were low, particularly when separated into BC subtypes.

Using ExoQuick on plasma, miR-16 was found at significantly higher levels in patients (24\% TNBC, 28\% ER ${ }^{+}$, HER2 status unknown) and those with ductal carcinoma in situ (DCIS) than in healthy controls. Specifically, EV miR-16 occurred at 2.1-fold ( $p$ $=0.034$ ) higher levels across all BCs when compared with controls. However, the EV miR-16 levels reported in DCIS (1.8-fold, $p$ $=0.047$ ) were not substantially different to BC [15]. This suggests that plasma-based miR-16 might have relevance as a diagnostic, even if the supporting data are not strong. However, further analysis of this data to consider BC subtypes showed that although plasma EV miR-16 levels were higher in $\mathrm{ER}^{+}$patients (1.5-fold, $p=0.004)$ compared with controls, the same did not hold true for TNBC patients. In this instance, miR-16 levels were lower in TNBC versus controls $(0.7$-fold, $p=0.012)$. This highlights the importance of considering each BC subtype independently when seeking diagnostic biomarkers; but to do so requires adequate numbers of samples representing each subtype.

Stevic et al. [16] also precipitated EVs from plasma using ExoQuick and characterised them by CD63-ELISA to consider EV concentrations in peripheral blood as diagnostic biomarkers. Here, higher concentrations of EVs in plasma from patients $(n=78$ HER $^{+}, p=0.0001 ; n=40$ TNBC, $p=0.002$ ) were reported in comparison to healthy controls $(n=10)$. Increasing the numbers of samples and performing miRNA profiling ( $n=384$ miRNAs) 
TABLE 2

Blood-based EV cargo proposed as potential diagnostic biomarkers in breast cancer

\begin{tabular}{llll}
\hline Patients & Controls & Biofluid & $\begin{array}{l}\text { EV quantities or } \\
\text { cargo proposed as } \\
\text { biomarker }\end{array}$
\end{tabular}

\section{RNAs}

EV concentration (by ExoQuick Immunoblot: CD63, CD81

[16]

\begin{tabular}{|c|c|}
\hline $\begin{array}{l}n=78 \text { HER2, } n=40 \\
\text { TNBC }\end{array}$ & $\begin{array}{l}n=10 \text { healthy } \\
\text { controls }\end{array}$ \\
\hline$n=44 \mathrm{BC}$ patients & $\begin{array}{l}n=12 \text { healthy } \\
\text { controls }\end{array}$ \\
\hline $\begin{array}{l}n=111 \mathrm{BC} \text { patients, } \\
n=42 \mathrm{DCIS} \text { patients }\end{array}$ & $\begin{array}{l}n=39 \text { healthy } \\
\text { controls }\end{array}$ \\
\hline $\begin{array}{l}n=179 \mathrm{BC} \text { patients } \\
\text { (incl. stages } 0 \text {-III) }\end{array}$ & $\begin{array}{l}n=20 \text { healthy } \\
\text { controls }\end{array}$ \\
\hline$n=16 \mathrm{BC}$ patients & $\begin{array}{l}n=16 \text { healthy } \\
\text { controls }\end{array}$ \\
\hline$n=46 \mathrm{BC}$ patients & $\begin{array}{l}n=28 \text { healthy } \\
\text { controls }\end{array}$ \\
\hline$n=32 \mathrm{BC}$ patients & $\begin{array}{l}n=32 \text { healthy } \\
\text { controls }\end{array}$ \\
\hline $\begin{array}{l}n=12 \text { early stage } \\
\text { (T1NOM0) BC }\end{array}$ & $\begin{array}{l}n=10 \text { controls with } \\
\text { benign breast } \\
\text { disease }\end{array}$ \\
\hline $\begin{array}{l}n=15 \text { BC pre- and } \\
\text { post-surgery }\end{array}$ & $\begin{array}{l}n=15 \text { healthy } \\
\text { controls }\end{array}$ \\
\hline
\end{tabular}

\section{Proteins}

$n=240$ early $\mathrm{BC}, n=$ $40 \mathrm{BC}$ patients postsurgery

$n=14$ BC patients, (i.
e., $n=7$ localised,
$n=7$ spread to
lymph nodes
$n=40$ BC patients
(stages I-IV)

$n=50 \mathrm{BC}$ patients (in

Other

$n=59 \mathrm{BC}$ positive individuals (mainly pools of samples studied) situ and stages I-III) $n=55$ benign breast disease, $n=80$ noncancer disease, $n=70$ disease-free individuals
Plasma

(1)

Plasma Periostin

$n=10$ controls, who had BC but diseasetreatment

$n=31$ healthy

controls

$n=30$ biopsy-

negative, $n=30$

healthy patients

(mainly pools of

samples studied)

Serum

$\Delta \mathrm{Ex} 3$

Plasma

Plasma

ssODNs free 5 years post-
ELISA-coated with

CD63 antibodies) and miRNAs

miR-101, $-372,-373$

miR-16

miR-223-3p

miR-1246, -21

miR-1246

miR-122-5p

miR-375, 24-2-5p,

548b-5p, 655-3p,

376-5p

HOTAIR

Fibronectin

Survivin, survivin-

EV concentration;

FAK, EGFR
ExoQuick

ExoQuick

Centrifugation

$(15,000 \mathrm{~g})$

ExoQuick

EV content evaluated

in situ in plasma

ExoQuick

ExoQuick

$0.45 \mu \mathrm{m}$ filtration; ExoQuick, $1500 \mathrm{~g}$ spin

Not isolated; captured by antiCD63 ELISA

UC (with a sucrose gradient centrifugation)

ExoQuick

UC

UC (with a sucrose gradient centrifugation)
Immunoblot: CD63, MUC1, GAPDH Immunoblot: $\mathrm{CD} 63, \mathrm{AGO}^{\mathrm{a}}$

TEM

Immunoblot: CD63, NTA; TEM

Immunoblot: CD63, TSG101; NTA; TEM

None

[19]

Immunoblot: CD9, TSG101, HSC70; TEM

Immunoblot: CD63, Hsp70; TEM

None

${ }^{a}$ AGO2, Argonaute RISC catalytic component 2; MHC-1, major histocompatibility complex 1; NTA, nanoparticle tracking analysis; TEM, transmission electron microscopy; TSG101, tumour susceptibility gene 101; UC, ultracentrifugation.

showed that 10 miRNAs were dysregulated in EVs from patients compared with controls, and other miRNA panels were associated with a BC subtype (HER2 or TNBC). Further analysis to potentially validate this in larger cohorts is necessary, although it should be considered that owing to the EV-isolation method used, it cannot be assumed that all miRNAs detected are EV cargo.

Yoshikawa et al. [17] used relatively low speed $(15,000 \mathrm{~g})$ centrifugation to separate EVs from the plasma of patients $[n=25$ DCIS; $n=94$ stage I, $n=52$ stage II and $n=8$ stage III invasive ductal carcinoma (IDC)], as well as $n=20$ healthy donors. It is noteworthy that this is likely to be a quite different population of EVs and other materials from those collected when using high speed (e.g., 110,000 g) centrifugation preparation styles. Here, levels of EV miR-223-3p significantly increased with progressing cancer stages (I-III) compared with stage 0 and controls. On average, EV miR-223-3p levels were 3.45-fold higher in IDC versus controls and 2.85-fold higher when compared with DCIS. Furthermore, EV miR-223-3p levels correlated significantly with nuclear grade and lymphatic invasion. However, no significant differences were found for BC subtypes. In this study, EV miR-16a was used as an internal control to normalise EV miR-223-3p [17]. Given that EV miR-16 had been reported as a potential biomarker distinguishing BC from healthy controls [15], this choice of control might require further consideration in future studies.

Using ExoQuick to precipitate EVs from the plasma of $\mathrm{BC}$ patients $\left(n=16\right.$, mostly $\mathrm{ER}^{+}$, grade $\left.2-3\right)$ and healthy controls 
$(n=16)$, Hannafon et al. [18] reported higher $(p<0.05)$ amounts of miR-1246 and miR-21 associated with cancer. This study was quite limited in sample size. However, following up on EV miR-1246 as a potential diagnostic, Zhai et al. [19] developed an Au nanoflare probe to detect EV-carried miR-1246 in situ in plasma. Without requiring $\mathrm{EV}$ isolation, the probe reportedly entered the plasma EVs and generated a quantitative fluorescent signal by targeting miR-1246. When evaluating plasma from patients $(n=46)$ and healthy controls $(n=28)$, the probe could discriminate the cancer samples as having significantly higher amounts of EV miR-1246 compared with that of the controls, with $100 \%$ sensitivity and up to $92.9 \%$ specificity. Clinical information such as BC subtype (or subtypes) was not detailed, and further research is necessary, including determining how and why the probe would specifically enter EVs, and conducting larger studies. However, the potential to quantify a specific miRNA as a component of plasma EV cargo without having to isolate the EVs makes this approach of much interest, providing that it continues to conform to being a potential diagnostic and that this technology can be adapted for clinical use.

As with many of these studies, Li et al. [20] used ExoQuick for EV precipitation from the plasma of BC patients and healthy controls $(n=32$ each). Of their five microRNAs of interest (miR-122-5p, miR-146-5p, miR-210-3p, miR-215-5p and let-7b-5p), miR-122-5p was found to be at higher $(p=0.001)$ levels in $\mathrm{BC}$. Whether or not all BC subtypes were represented was not clarified.

Although the population sample sizes from which EVs were precipitated using ExoQuick by Yan et al. [21] were very small (i. e., $n=12 \mathrm{BC}$ and $n=10$ from controls with benign breast disease), the fact that the BC patients were all early stage (T1NOM0) makes this a useful cohort when trying to identify early diagnostic biomarkers. Using sequencing, five miRNAs were identified that synchronised in EVs and BC tissue. Of those, miR-375 and miR-24-2-5p were negatively associated with patients' survival, whereas miRNA-548b-5p and miR-376-5p were positively associated with survival. Follow-up by qPCR with larger cohorts is necessary.

Notwithstanding a few exceptions such as miR-1246, it is noteworthy that most part of the miRNAs identified as potential diagnostic biomarkers differ between the discovery studies mentioned above. The reasons for this could include different, relatively small, donor cohorts; different pre-analytical steps before plasma or sera separation; some studies using plasma and others using sera; different methods for EV isolation (if EVs were isolated); and differences in characterisation to determine whether the isolates were EVs before proceeding to miRNA analysis. In addition, the miRNA analysis method differed from study to study, with methods including qPCR (Taqman or Maxima SYBR Green), qPCR-based microarrays (with one such study using arrays representing 384 miRNAs and another study using arrays representing only 45 different miRNAs) and miRNA sequencing using an Au nanoflare probe in situ. Of note, some of these methods are not truly quantitative and thus, as performed, are incomparable between studies. Differences also occurred in the analysis of the resulting data, which were sometimes normalised, and sometimes not. Considering all these differences, it is probably unsurprising that there were only few agreements between the study outcomes in terms of the specific miRNAs identified as potential diagnostics.
However, collectively, the studies suggest that some miRNAs might have potential as EV-carried biomarkers. Advancing on these preliminary studies, it would be important to develop standard operating procedures (SOPs) for each step involved and to perform larger (ideally, multi-centre) studies to conclusively determine whether EV miRNAs can be analysed reliably, robustly and reproducibly as diagnostic biomarkers for BC.

MiRNAs are not the only EV cargo that has potential as diagnostic biomarkers. Other RNAs, such as the long non-coding RNA (lncRNA) HOX transcript antisense RNA (HOTAIR), have also been proposed for this role. Specifically, using filtration and ExoQuick to precipitate EVs from serum, Tang et al. [22] reported EV-carried HOTAIR to be at higher $(p<0.001)$ levels in sera from BC patients $(n=15)$ compared with healthy controls $(n=15)$. Furthermore, three months post-surgery, the EV HOTAIR levels in the patient cohorts' sera had fallen significantly $(p<0.001)$, supporting the hypothesis that it originated from tumours.

Proteins can also be used as diagnostic biomarkers. For example, fibronectin is an essential extracellular matrix protein that is proposed to facilitate cancer invasion and metastasis [23,24], and EV-carried fibronectin was reported [25] to have greater diagnostic accuracy than fibronectin analysed directly in plasma. In this study, a form of in-house sandwich ELISA was used in which plasma was incubated on 96-well plates coated with polyclonal anti-CD63 antibody. The excess was washed off and then reacted with an anti-fibronectin monoclonal antibody. Notwithstanding the fact that very small volumes $(1 \mu \mathrm{l})$ of plasma were required, it is important to consider that EVs were not isolated and thus were not definitively characterised as EVs. The study represents a potentially useful way to analyse plasma biomarkers in a clinical setting in which limited volumes of biofluid might be available, but there is no substantial evidence that the fibronectin analysed was EVbased.

Periostin is another major component of the extracellular matrix that is upregulated in cancer [26,27], and it is enriched in EVs secreted from metastatic BC cells [28]. Vardaki et al. [28] isolated EVs using ultracentrifugation from the plasma of BC patients whose cancer had remained localised $(n=7)$ or spread to the lymph nodes (LNs) $(n=7)$, and they found that EV-carried periostin was typically at a higher level in patients with LN involvement, although they did not evaluate EVs for cancer diagnosis per se. Owing to the low number of samples and the fact that not all $\mathrm{LN}^{-}$cases had an absence of $\mathrm{EV}$ periostin and not all $\mathrm{LN}^{+}$cases had EV periostin present, it is impossible to draw clinically meaningful conclusions. However, if $\mathrm{EV}$ periostin could predict which tumours would remain localised rather than spreading to the LNs, this could help to avoid unnecessary treatment for those who do not need it, while prioritising those who do. A higherthroughput and quantitative method, rather than immunoblotting as used here, would be necessary for clinical use. These researchers also reported that the EVs/particles from the plasma of those who were $\mathrm{LN}^{+}$, compared with those who were $\mathrm{LN}^{-}$, were larger (on average, $125 \mathrm{~nm}$ versus $95 \mathrm{~nm}$ ) and at a higher concentration (on average, $10.9 \times 10^{9}$ per $\mathrm{ml}$ versus $8.35 \times 10^{9}$ per $\mathrm{ml}$ ), based on nanoparticle tracking analysis. However, despite an observed difference in EV concentrations, for unexplained reasons, a fixed quantity of EV protein from each sample was used for comparative periostin immunoblotting. 
We and others have reported that anti-apoptotic survivin is overexpressed in BC $[29,30]$. Khan et al. [31] sought to investigate the early diagnostic value of survivin and its splice variants by immunoblotting. They recruited a cohort of BC patients (stages IIV) and used ExoQuick to precipitate EVs from serum, then evaluated acetylcholinesterase activity (as an EV-associated enzyme), although unusually the controls $(n=10)$ used were sera from former cancer patients. This study showed that acetylcholinesterase activity $(p<0.01)$, survivin and survivin- $\Delta \mathrm{Ex} 3$ were higher (the latter two averaging $\sim 1$.4-fold higher) in cancer compared with controls. However, as we have mentioned in Table 2, acetylcholinesterase has recently been reported not to be a generic marker of EVs [32]. Relevant future studies could use larger cohorts, with consideration given to the most appropriate controls, as well as fundamental EV characterisation and higherthroughput, more quantitative methods (such as ELISA) for survivin analysis.

Galindo-Hernandez et al. [33] investigated focal adhesion kinase (FAK) and epidermal growth factor receptor (EGFR). FAK correlates with increased invasion and metastasis in BC [33]. Here, EVs were isolated from platelet-deprived/platelet-poor plasma of $\mathrm{BC}$ patients $(n=50)$ and healthy controls $(n=31)$ using differential ultracentrifugation. Although the main focus was not EV concentrations, significantly higher concentrations of EVs $(p<0.0001)$ were reported for the patients' cohort (on average, $\sim 750 \mathrm{EVs} / \mu \mathrm{l}$ ) compared with the control cohort (on average, $\sim 300 \mathrm{EVs} / \mu \mathrm{l}$ ). Within the patients' cohort, the EV concentrations were significantly greater for those with stage I $(n=9)$, stage II $(n=23)$, stage III $(n=12)$ and stage IV $(n=3)$ BC compared with in situ carcinoma $(n=3)$, although the sample sizes per group were small. Appropriately, using equal volumes of plasma fractions enriched in EVs (rather than fixed protein quantities, which would lose the significance of more EVs with cancer), immunoblot analysis of
EV-carried FAK and EGFR showed that both proteins were absent from the control samples. However, FAK was detected in EVs from those with BC in situ and stage I-III BC, with increasing FAK associated with increasing stages. EV EGFR, conversely, was at highest levels in in situ cases, and decreased with increasing stages.

Adaptive dynamic artificial poly-ligand targeting (ADAPT) has been used to profile thousands of single-stranded oligodeoxynucleotides (ssODNs), analysing EVs from pooled plasma samples taken from patients whose biopsies were positive or negative, or from healthy controls. Domenyuk et al. [34] reported that a subset of 2000 ssODNs could stratify healthy from breast biopsy-negative and -positive women. With further refinement and validation, this approach might enable cross-referencing of an uncharacterised sample against known biomolecular signatures to diagnose BC. However, whether such a complex approach could be advanced to routine clinical use has yet to be considered. Its strength might be in the discovery of biomarkers, which could then be validated and detected using a simpler method.

\section{EVs as predictive biomarkers}

Predictive biomarkers can forecast whether an individual will benefit from a particular treatment regime [2]. In an effort to establish the potential of EVs to predict response to pharmacological intervention (Table 3), Chen et al. [35] isolated EVs via ultracentrifugation from plasma procured during anthracycline-taxane-based adjuvant chemotherapy treatment $(n=34)$, and also from patients who received no chemotherapy post-surgery $(n=21)$. Breast cancer resistance protein (BCRP), flotillin 2 and mucin 1 (MUC1) mRNAs were at significantly higher levels in non-responders compared with those who did not receive adjuvant chemotherapy. Furthermore, EVs from non-responders had higher MUC1 protein levels (evaluated by immunofluorescence), often co-expressed with BCRP. BCRP was mostly absent for those who received no treatment. The study

TABLE 3

\begin{tabular}{|c|c|c|c|c|c|}
\hline Cohorts & Biofluid & $\begin{array}{l}\text { EV concentration or } \\
\text { cargo proposed as } \\
\text { biomarker }\end{array}$ & EV isolation method & $\begin{array}{l}\text { EV characterisation } \\
\text { performed }\end{array}$ & Refs \\
\hline $\begin{array}{l}n=34 \text { adjuvant } \mathrm{TEC}^{\mathrm{a}}, n=21 \text { receiving no } \\
\text { chemotherapy }\end{array}$ & Plasma & BCRP & Differential UC & None & [35] \\
\hline$n=131$ unresectable metastases & Plasma & TRPC5 & Differential UC & $\begin{array}{l}\text { Immunoblot: CD63, } \\
\text { flotillin 1, MUC1; } \\
\text { immunofluorescence: } \\
\text { CD63, MUC1 }\end{array}$ & {$[36]$} \\
\hline $\begin{array}{l}n=30 \text { HER2 neo-adjuvant to trastuzumab } \\
\text { and lapatinib [NCT01485926] }\end{array}$ & Serum & TGF $\beta_{1}$ & Differential UC & $\begin{array}{l}\text { Immunoblots: CD9, } \\
\text { CD63, CD81, Alix, GRP94; } \\
\text { NTA; TEM }\end{array}$ & [37] \\
\hline $\begin{array}{l}n=38 \mathrm{HER}^{+} \text {trastuzumab responsive, } \\
n=34 \mathrm{HER}^{+} \text {trastuzumab resistance }\end{array}$ & Serum & IncRNA SNHG14 & ExoQuick & $\begin{array}{l}\text { Immunoblots: CD9, } \\
\text { CD63, CD81, Alix; NTA; } \\
\text { TEM }\end{array}$ & {$[38]$} \\
\hline $\begin{array}{l}n=435 \text { pre-therapy ( } n=211 \text { HER } 2, n=224 \\
\text { TNBC) to neo-adjuvant paclitaxel } \\
+ \text { doxorubicin }+/- \text { carboplatin }\end{array}$ & Plasma & $\begin{array}{l}\text { EV concentration (by } \\
\text { ELISA-coated with } \\
\text { CD63 antibodies) }\end{array}$ & ExoQuick & $\begin{array}{l}\text { Immunoblot: CD63, } \\
\text { CD81 }\end{array}$ & [16] \\
\hline $\begin{array}{l}n=40 \mathrm{HR}^{+} / \mathrm{HER} 2^{-} \text {advanced breast cancer } \\
\text { prior to palbociclib }\end{array}$ & Plasma & TK1, CDK9 & exoRNeasy & None & [39] \\
\hline $\begin{array}{l}n=25 \text { neo-adjuvant chemotherapy, } n=25 \\
\text { neo-adjuvant tamoxifen }\end{array}$ & Serum & HOTAIR & $\begin{array}{l}\text { Filtration ( } 0.45 \mu \mathrm{m} \text { filter), } \\
\text { then ExoQuick, with } \\
1500 \mathrm{~g} \text { spin }\end{array}$ & $\begin{array}{l}\text { Immunoblot: CD63, } \\
\text { Hsp70; TEM }\end{array}$ & {$[22]$} \\
\hline
\end{tabular}

${ }^{a}$ NTA, nanoparticle tracking analysis; TEC, anthracycline-taxane-based chemotherapy; TEM, transmission electron microscopy; UC, ultracentrifugation. 
concluded that EV-carried BCRP might predict response to chemotherapy. Of note, those who received chemotherapy tended to have more advanced tumours and LN involvement, compared with those who did not, so the fact that their EVs carry more BCRP is probably not unexpected.

Wang et al. [36] used ultracentrifugation to isolate EVs from plasma taken from patients with unresectable metastases before treatment. They showed that those who went on to respond to the anthracycline/taxane-based chemotherapy had significantly lower levels of EV-carried transient receptor potential channel 5 (TRPC5) compared with non-responders (the range of EV TRPC5 was 3.1-88.6; lower and higher levels were defined by the median, with the values being arbitrary immunofluorescence values, as measured by flow cytometry). A $>1.09$-fold increase in EV TRPC5 after two cycles of chemotherapy (which occurred for $n=36$ patients, but not for $n=52$ patients) was associated ( $p=0.0042$ ) with reduced progressionfree survival. These results are interesting, but because the cutoff thresholds were fitted retrospectively, it would be important to see how this holds out in prospective studies. It remains unclear how practical a 1.09-fold difference might be for predicting a response.

Our group collected serum before neoadjuvant treatment with HER2-targeted drugs (trastuzumab and lapatinib) in a Phase I clinical trial ( $n=30$ patients; NCT01485926). Following EV isolation by ultracentrifugation, we discovered that levels of transforming growth factor- $\beta_{1}$ (TGF $\left.\beta_{1}\right)$, which tethered to the surface of EVs and suppressed immune cells, were higher $(p<0.01)$ for patients who subsequently did not gain benefit from the treatment compared with those who did [37]. This suggests EV-carried TGF $\beta_{1}$ as a predictive biomarker for some HER2-targeted drugs. Following this, other researchers procured pre-treatment serum from advanced HER $2^{+}$patients $(n=72)$, who then received trastuzumab. Serum precipitates analysed following ExoQuick isolation showed lower amounts of long non-coding small nucleolar RNA host gene 14 (lncSNHG14) $(p=0.025)$ and total SNHG14 $(p=0.0139)$ in responders $(n=38)$ compared with non-responders $(n=34)$. Furthermore, higher levels of SNHG14 were associated $(p<0.01)$ with $\mathrm{LN}^{+}$cancer, distant metastasis and cardiac toxicity [38].

After profiling 435 plasma samples precipitated by ExoQuick, Stevic et al. [16] reported that miR-155 $(p=0.002)$ and miR-301 $(p=0.002)$ could predict pathological complete response to neoadjuvant paclitaxel + doxorubicin +/- carboplatin. Efforts to validate these findings by qPCR, ideally using EV isolation methods that would give confidence that the miRNAs are carried by EVs, would be welcome.

Del Re et al. [39] analysed plasma from patients with advanced $\mathrm{BC}\left(n=40, \mathrm{HER}^{+} / \mathrm{HER}^{-}\right)$before treatment with the cyclin-dependent kinase 4 (CDK4) and CDK6 inhibitor palbociclib and hormone therapy. EVs were not characterised. ExoRNeasy was used to obtain RNA, which was analysed by digital-droplet PCR. High baseline CDK4 was associated ( $p=0.01)$ with increased progression-free survival (taking 6.45 months as a cut-off time point) in patients treated with palbociclib and fulvestrant. Conversely, increasing levels of thymidine kinase 1 (TK1) and CDK9 mRNA over the course of treatment were associated with drug-resistance. This finding warrants further evaluation in larger cohorts, but because EVs were not characterised, it is uncertain whether the mRNAs studied were specifically EV-associated. Furthermore, an explanation for the statistical analysis at 6.45 months post-treatment would have been welcome.

After EV precipitation (by filtration and ExoQuick) from the sera of two cohorts of patients $(n=25$ each) prior to neo-adjuvant chemotherapy and tamoxifen, respectively, Tang et al. [22] reported that relatively high levels of EV-HOTAIR before treatment might be associated with poor response to both treatment regimes. Again, independent validation in larger cohorts and with methods of isolation and characterisation that can support the EV nature of this RNA would be important.

\section{Concluding remarks}

As reviewed, substantial efforts are underway to identify bloodbased diagnostic and predictive biomarkers for BC. Each study suggests that the RNAs or proteins being analysed are EV cargo. However, some do not separate EVs from the plasma/serum (although this lack of separation step might be ideal for ease of use clinically, if these assays can be validated); some precipitate EVs along with RNAs and proteins that are not EV cargo; and some assume the presence of EVs, rather than characterise them. To avoid confusion, ideally researchers should avoid claiming their analysis is specifically of EVs if there is no support for this. Thus, although caution should be given when claiming that a biomarker originates from an EV, if an RNA or protein can be validated as a reliable blood-based diagnostic or predictive biomarker, then from a clinical perspective the matter of whether or not it is an EV cargo is a secondary concern.

Good preliminary data have been generated, but more research is needed. We recommend considering the MISEV2018 guidelines [8] to be confident of working with EVs, if that is what is intended. We urge that consideration should be given to preanalytical steps in sample preparation, prospective studies, analyses of large cohorts of specimens (keeping in mind BC subtypes and the male cohort) with appropriate controls, using methods that could be translated to clinical use, and concentrating on markers that are different enough between the groups being considered so that they could be analysed with reliability in the clinical setting. Building on previous achievements, such efforts could realistically result in new diagnostic and predictive biomarkers for BC. In fact, some molecules might have potential as both diagnostics and predictive biomarkers. However, this cannot be assumed, and so robust evaluation is needed to investigate whether this is so.

Future considerations of EVs in blood could give consideration to reviews such as that by Monguio-Torajada et al. [40], which qualitatively summarises ten different $\mathrm{EV}$ isolation techniques based on 12 different criteria, albeit not specifically related to BC. Furthermore, future studies comparing serum and plasma from the same BC patients would be needed to properly determine which is superior as a source of EVs when evaluating potential diagnostic and/or predictive biomarkers. However, it must also be considered that this would require twice as much blood to be taken to separate as both serum and plasma. Although there might be no ethical concerns in seeking consent from healthy volunteers to donate larger volumes of blood, for already ill cancer patients, who are probably having multiple blood samples taken for standard-of-care tests, this 
might not be possible. As mentioned earlier, in efforts to move towards greater rigour and standardisation, it is important for researchers with an interest in this area to work together to develop SOPs for each step, including how patients are initially asked to present (e.g., in the fasting state) to have their blood samples taken; how the samples are processed as serum or plasma; how samples are stored and shipped (if relevant); how EVs are isolated and characterised; how EV numbers and/or cargo are analysed and then validated; and how the resulting data are analysed and interpreted. Ultimately, well-controlled, large, blinded, multi-centre studies are required to definitively establish whether EVs are viable as diagnostic and predictive tools in $\mathrm{BC}$.

\section{Conflict of interest statement}

The authors have no conflicts of interest to declare.

\section{Acknowledgements}

This work was supported by an Irish Research Council Advanced Laureate Award to L.O.D. as principal investigator, and on which R.D. is a PhD student [IRCLA/2019/49].

\section{References}

1 American Cancer Society (2020) Cancer Facts \& Figures 2020. American Cancer Society

2 Califf, R.M. (2018) Biomarker definitions and their applications. Exp. Biol.Med. 243, 213-221

3 Fox, A.S. et al. (1970) DNA-induced transformation in Drosophila: evidence for transmission without integration. Proc. Natl. Acad. Sci. U. S. A. 67, 1834-1838

4 Johnstone, R.M. et al. (1987) Vesicle formation during reticulocyte maturation. Association of plasma membrane activities with released vesicles (exosomes). J. Biol. Chem. 262, 9412-9420

5 van der Pol, E. et al. (2012) Classification, functions, and clinical relevance of extracellular vesicles. Pharmacol. Rev. 64, 676-705

6 Yáñez-Mó, M. et al. (2015) Biological properties of extracellular vesicles and their physiological functions. J. Extracell. Vesicles 4, 27066

7 Pegtel, D.M. and Gould, S.J. (2019) Exosomes. Annu. Rev. Biochem. 88, 487-514

8 Thery, C. et al. (2018) Minimal information for studies of extracellular vesicles 2018 (MISEV2018): a position statement of the International Society for Extracellular Vesicles and update of the MISEV2014 guidelines. J. Extracell. Vesicles 7, 1535750

9 Gardiner, C. et al. (2016) Techniques used for the isolation and characterization of extracellular vesicles: results of a worldwide survey. J. Extracell. Vesicles 5, 32945

10 Van Deun, J. et al. (2014) The impact of disparate isolation methods for extracellular vesicles on downstream RNA profiling. J. Extracell. Vesicles 3, 24858

11 Lobb, R.J. et al. (2015) Optimized exosome isolation protocol for cell culture supernatant and human plasma. J. Extracell. Vesicles 4, 27031

12 Karimi, N. et al. (2018) Detailed analysis of the plasma extracellular vesicle proteome after separation from lipoproteins. Cell Mol. Life Sci. 75, 2873-2886

13 Clayton, A. et al. (2019) Considerations towards a roadmap for collection, handling and storage of blood extracellular vesicles. J. Extracell. Vesicles 8, 1647027

14 Eichelser, C. et al. (2014) Increased serum levels of circulating exosomal microRNA373 in receptor-negative breast cancer patients. Oncotarget 5, 9650-9663

$15 \mathrm{Ni}$, Q. et al. (2018) Different signatures of miR-16, miR-30b and miR-93 in exosomes from breast cancer and DCIS patients. Sci. Rep. 8, 12974

16 Stevic, I. et al. (2018) Specific microRNA signatures in exosomes of triple-negative and HER2-positive breast cancer patients undergoing neoadjuvant therapy within the GeparSixto trial. BMC Med. 16, 179

17 Yoshikawa, M. et al. (2018) Exosome-encapsulated microRNA-223-3p as a minimally invasive biomarker for the early detection of invasive breast cancer. Oncol. Lett. 15, 9584-9592

18 Hannafon, B.N. et al. (2016) Plasma exosome microRNAs are indicative of breast cancer. Breast Cancer Res. 18, 90

19 Zhai, L.Y. et al. (2018) In situ detection of plasma exosomal microRNA-1246 for breast cancer diagnostics by a Au nanoflare probe. ACS Appl. Mater Interfaces 10, 39478-39486

20 Li, M. et al. (2019) A five-miRNA panel in plasma was identified for breast cancer diagnosis. Cancer Med. 8, 7006-7017

21 Yan, C. et al. (2019) Plasma extracellular vesicle packaged microRNAs as candidate diagnostic biomarkers for early stage breast cancer. Mol. Med Rep. 20 3991-4002
22 Tang, S. et al. (2019) Overexpression of serum exosomal HOTAIR is correlated with poor survival and poor response to chemotherapy in breast cancer patients. J. Biosci. 44,37

23 Ohnishi, T. et al. (1998) Role of fibronectin-stimulated tumor cell migration in glioma invasion in vivo: clinical significance of fibronectin and fibronectin receptor expressed in human glioma tissues. Clin. Exp. Metastasis 16, 729-741

24 Manabe, R. et al. (1999) Alternatively spliced EDA segment regulates fibronectindependent cell cycle progression and mitogenic signal transduction. J. Biol. Chem. 274, 5919-5924

25 Moon, P.G. et al. (2016) Fibronectin on circulating extracellular vesicles as a liquid biopsy to detect breast cancer. Oncotarget 7, 40189-40199

26 Gillan, L. et al. (2002) Periostin secreted by epithelial ovarian carcinoma is a ligand for $\alpha_{V} \beta_{3}$ and $\alpha_{V} \beta_{5}$ integrins and promotes cell motility. Cancer Res. 62, 5358-5364

27 Michaylira, C.Z. et al. (2010) Periostin, a cell adhesion molecule, facilitates invasion in the tumor microenvironment and annotates a novel tumor-invasive signature in esophageal cancer. Cancer Res. 70, 5281-5292

28 Vardaki, I. et al. (2016) Periostin is identified as a putative metastatic marker in breast cancer-derived exosomes. Oncotarget 7, 74966-74978

29 Kennedy, S.M. et al. (2003) Prognostic importance of survivin in breast cancer. Br. J. Cancer 88, 1077-1083

30 O'Driscoll, L. et al. (2003) Survivin: role in normal cells and in pathological conditions. Curr. Cancer Drug Targets 3, 131-152

31 Khan, S. et al. (2014) Early diagnostic value of survivin and its alternative splice variants in breast cancer. BMC Cancer 14, 176

32 Liao, Z. et al. (2019) Acetylcholinesterase is not a generic marker of extracellular vesicles. J. Extracell. Vesicles 8, 1628592

33 Galindo-Hernandez, O. et al. (2013) Elevated concentration of microvesicles isolated from peripheral blood in breast cancer patients. Arch. Med. Res. 44, 208-214

34 Domenyuk, V. et al. (2017) Plasma exosome profiling of cancer patients by a next generation systems biology approach. Sci. Rep. 7, 42741

35 Chen, Y. et al. (2015) Breast cancer resistance protein (BCRP)-containing circulating microvesicles contribute to chemoresistance in breast cancer. Oncol. Lett. 10, 3742 3748

36 Wang, T. et al. (2017) Increasing circulating exosomes-carrying TRPC5 predicts chemoresistance in metastatic breast cancer patients. Cancer Sci. 108, 448-454

37 Martinez, V.G. et al. (2017) Resistance to HER2-targeted anti-cancer drugs is associated with immune evasion in cancer cells and their derived extracellular vesicles. Oncoimmunology 6, e1362530

38 Dong, H. et al. (2018) Exosome-mediated transfer of lncRNASNHG14 promotes trastuzumab chemoresistance in breast cancer. Int. J. Oncol. 53, 1013-1026

39 Del Re, M. et al. (2019) Overexpression of TK1 and CDK9 in plasma-derived exosomes is associated with clinical resistance to CDK4/6 inhibitors in metastatic breast cancer patients. Breast Cancer Res. Treat. 178, 57-62

40 Monguió-Tortajada, M. et al. (2019) Extracellular vesicle isolation methods: rising impact of size-exclusion chromatography. Cell Mol. Life Sci. 76, 2369-2382 